
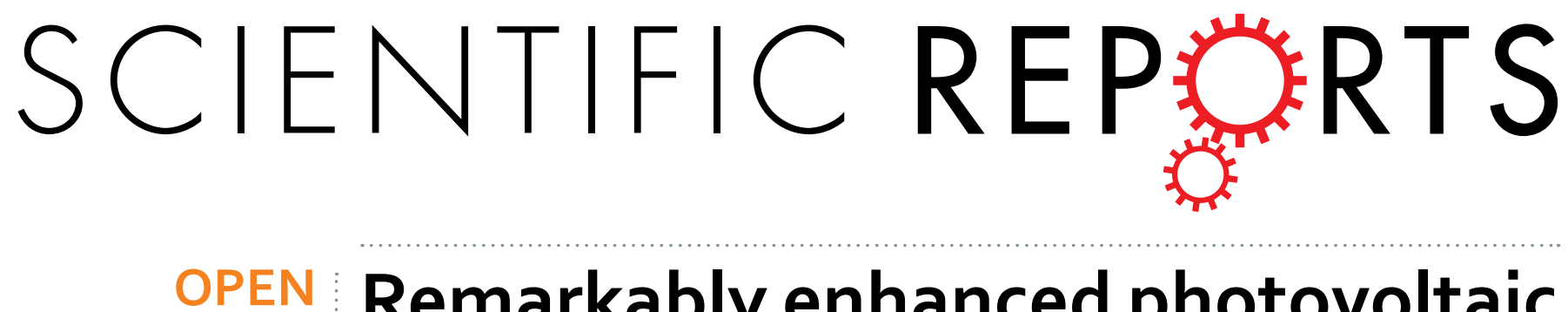

\title{
Remarkably enhanced photovoltaic effects and first-principles calculations in neodymium doped \\ $\mathrm{BiFeO}_{3}$
}

Received: 11 October 2016

Accepted: 20 February 2017

Published: 24 March 2017

\author{
Yi-Ting Peng ${ }^{1}$, Shan-Haw $\mathrm{Chiou}^{2}$, Ching-Hung Hsiao ${ }^{1}$, Chuenhou (Hao) Ouyang ${ }^{1}$ \& \\ Chi-Shun Tu ${ }^{3}$
}

Remarkably enhanced photovoltaic effects have been observed in the heterostructures of $p$-type A-site $\mathrm{Nd}^{3+}$-doped $\mathrm{BiFeO}_{3}\left(\mathrm{Bi}_{0.9375} \mathrm{Nd}_{0.0625}\right) \mathrm{FeO}_{3}$ (or $\mathrm{BFONd}$ ) polycrystalline ceramics and the $\mathrm{n}$-type ITO thin film. The maximum power conversion is $\sim 0.82 \%$, which is larger than $0.015 \%$ in $\mathrm{BiFeO}_{3}(\mathrm{BFO})$ under blue-ultraviolet irradiation of wavelength $\lambda=405 \mathrm{~nm}$. The current-voltage (I-V) characteristic curve suggests a p-n junction interface between the ITO thin film and BFO (or BFONd) ceramics. The band gaps calculated from first-principles for BFO and BFONd are respectively $2.25 \mathrm{eV}$ and $2.23 \mathrm{eV}$, which are consistent with the experimental direct band gaps of $2.24 \mathrm{eV}$ and $2.20 \mathrm{eV}$ measured by optical transmission spectra. The reduction of the band gap in BFONd can be explained by the lower electronic Fermi level due to acceptor states revealed by first-principles calculations. The optical calculations show a larger absorption coefficient in BFONd than in BFO.

Multiferroic $\mathrm{BiFeO}_{3}(\mathrm{BFO})$ possesses a ferroelectric Curie temperature of $\mathrm{T}_{\mathrm{c}} \sim 1103 \mathrm{~K}$ and a G-type antiferromagnetic order (Neel temperature $\mathrm{T}_{\mathrm{N}} \sim 643 \mathrm{~K}$ ) with a spatially modulated spin structure. There is also the existence of weak Dzyaloshinskii-Moriya-type ferromagnetism ${ }^{1}$. Ferroelectrics have recently received lots of interest for potential applications in photovoltaic $(\mathrm{PV})$ responses $^{2}$. The major advantage of BFO is a relatively small band gap $(2.2 \sim 2.8 \mathrm{eV})^{3-9}$ in the visible spectrum amongst the other ferroelectric oxides with large band gaps $(>3 \mathrm{eV})$, including $\mathrm{LiNbO}_{3}, \mathrm{BaTiO}_{3}, \mathrm{SrTiO}_{3}$, and $\mathrm{Pb}(\mathrm{Zr}, \mathrm{Ti}) \mathrm{O}_{3}{ }^{3,10}$. Several mechanisms of photovoltaic effects have been proposed for BFO thin films and crystals, including the bulk photovoltaic effect ${ }^{11}$, the domain-wall model ${ }^{12}$, and lastly, the semiconductor-like p-n junction $\operatorname{model}^{4}$, the one adopted in this study. Our recent studies suggested that domain structure and hybridization between the $\mathrm{O} 2 p$ and $\mathrm{Fe} 3 d$ orbitals play important roles for the PV responses in the $\mathrm{ITO} /\left(\mathrm{Bi}_{1-x} \mathrm{Sm}_{x}\right) \mathrm{FeO}_{3}$ ceramic/Au heterostructure ${ }^{13}$.

$\mathrm{BFO}$ thin film has been reported as a p-type semiconductor, revealed by the rectifying current density versus voltage $(\mathrm{J}-\mathrm{V})$ behavior due to Bi deficiency during the high-temperature sintering process ${ }^{14}$. The first-principles calculation found that $\mathrm{Bi}$ vacancies have lower defect formation energy than oxygen vacancies, suggesting that $\mathrm{Bi}$ vacancies are the acceptor defects that cause $\mathrm{BFO}$ to become a p-type semiconductor ${ }^{15}$. However, BFO exhibited serious current leakage due to oxygen vacancies causing a valence shift of $\mathrm{Fe}^{3+} \rightarrow \mathrm{Fe}^{2+16}$ that formed shallow energy centers (defect states) ${ }^{17}$. This is a problem in the industrial application of photovoltaic devices. The density-functional-theory (DFT) calculation and X-ray absorption spectroscopy revealed that the A-site rare-earth lanthanum (La) doping in BFO can reduce the leakage current ${ }^{18}$. It has been reported that doping can be one of the most effective methods used to improve ferroelectric and magnetic properties ${ }^{19-22}$, as the Bi-site substitution can effectively control the volatility of $\mathrm{Bi}$ atoms with reduction of oxygen vacancies ${ }^{23}$ and enhancement of photovoltaic effects ${ }^{24-26}$. For photovoltaic applications, the BFO should be able to absorb as much light as possible to generate a photocurrent, which requires a lower band gap and large absorption coefficient ${ }^{27}$. The photocurrent density under irradiation with a certain wavelength can be described by an empirical Glass Law ${ }^{28}$ :

${ }^{1}$ Department of Materials Science and Engineering, National Tsing Hua University, Hsinchu, Taiwan 300, Republic of China. ${ }^{2}$ Material and Chemical Research Laboratories and Nanotechnology Research Center, Industrial Technology Research Institute, Hsinchu, Taiwan 310, Republic of China. ${ }^{3}$ Department of Physics, Fu Jen Catholic University, New Taipei City, Taiwan 24205, Republic of China. Correspondence and requests for materials should be addressed to C.O. (email: houyang@mx.nthu.edu.tw) or C.-S.T. (email: chishun.tu@gmail.com) 


$$
J_{\mathrm{ph}}=\alpha \kappa \mathrm{I}
$$

where $\alpha$ is the optical absorption coefficient, $\kappa$ is a material-dependent Glass coefficient, and I is irradiation intensity. The photocurrent density $\mathrm{J}_{\mathrm{ph}}$ is linearly proportional to the absorption coefficient. The relation between $\alpha$ and an optical band gap $E_{\mathrm{g}}$ can be estimated using the Tauc relation, i.e..$^{5,29}$

$$
\alpha(E) \sim \mathrm{A}\left(E-E_{\mathrm{g}}\right)^{1 / 2}
$$

where $\mathrm{A}$ is a material-dependent constant and $E$ is the incident photon energy. According to Eqs (1) and (2), one can extrapolate a larger photocurrent density by increasing the absorption coefficient with a small band gap.

In the study, enhanced photovoltaic effects have been observed in the ITO/BFONd ceramic/Au heterostructure under blue-ultraviolet irradiation of $\lambda=405 \mathrm{~nm}$, where the power conversion efficiency reached $\sim 0.82 \%$, higher than that of most BFO materials ${ }^{13,30-34}$. It opens promising future applications for optical devices such as photo-driven actuators and optical sensors. The first-principles calculation suggests that the reduction of the band gap resulted from a lower electronic Fermi level responsible for the enhanced photovoltaic responses. The calculated band gaps of $2.25 \mathrm{eV}$ (for BFO) and $2.23 \mathrm{eV}$ (for BFONd) are thus consistent with experimental values of $\sim 2.24 \mathrm{eV}$ and $\sim 2.20 \mathrm{eV}$, respectively.

\section{Experimental}

$\left(\mathrm{Bi}_{0.9375} \mathrm{Nd}_{0.0625}\right) \mathrm{FeO}_{3}$ (BFONd) ceramics were prepared using the solid state reaction method, in which $\mathrm{Bi}_{2} \mathrm{O}_{3}$, $\mathrm{Nd}_{2} \mathrm{O}_{3}$, and $\mathrm{Fe}_{2} \mathrm{O}_{3}$ powders (purity $\geq 99.0 \%$ ) were weighed in a ratio of 0.95:0.05:1.0. The powders were mixed in an agate mortar for more than 24 hours, with alcohol as a medium. The dried mixtures were calcined at $800^{\circ} \mathrm{C}$ for 3 hours and then sintered at $870^{\circ} \mathrm{C}$ for three hours. X-ray diffraction (XRD) spectra were performed using a Shimadzu-XRD6000 X-ray diffractometer. The Rietveld-refinement method, used in the General Structure Analysis System (GSAS), was performed for crystallographic analysis and the determination of $\mathrm{Nd}$ concentration ${ }^{35}$. Scanning transmission electron microscopy (STEM) imaging was performed using Cs corrected-field-emission TEM (Ultra-HRTEM). For photovoltaic (PV) measurements, ITO ( 100 nm) and Au thin films were deposited on the ceramic surfaces by dc sputtering as top and bottom electrodes respectively. The irradiated area (also known as the electrodes area) of the samples is about $0.15 \mathrm{~cm}^{2}$. A diode laser $(\lambda=405 \mathrm{~nm})$ was used as the illumination source, and the laser beam was incident perpendicular to the sample surface with the ITO film.

\section{Calculations}

$\mathrm{Ab}$ initio calculations were performed using the accurate full-potential projector augmented wave (PAW) method $^{36}$ as implemented in the Vienna ab initio simulation package (VASP) $)^{37,38}$. Our first-principles approach is based on the LSDA+U method ${ }^{39,40}$ with a Hubbard parameter from $5.0 \mathrm{eV}$ to $9.0 \mathrm{eV}$ and an effective on-site exchange interaction of $\mathrm{J}=0.9 \mathrm{eV}$ for Fe $3 d$ electrons ${ }^{41}$. A large plane-wave cutoff of $500 \mathrm{eV}^{41}$ was used throughout the calculation. Brillouin zone integrations were performed with the tetrahedron method, using Blöchl corrections in a $3 \times 3 \times 3$ Monkhorst-Pack k-point mesh centered at $\Gamma^{42}$ and the k-points mesh was $10 \times 10 \times 10$ for density of states (DOS) calculations. The unit cell of rhombohedral $R 3 c$ space group for $\mathrm{BiFeO}_{3}$ was extended to a $2 \times 2 \times 2$ super ell containing 80 atoms which includes 16 bismuth atoms, 16 iron atoms and 48 oxygen atoms to simulate the $\mathrm{Nd}$ doping atomic ratio. All structures were full relaxed. The formation energy calculation was performed with one $\mathrm{Nd}$ atom substitutes doping the $\mathrm{A}(\mathrm{Bi})$-site, or $\mathrm{Fe}$-site, or interstice $\mathrm{B}, \mathrm{C}$ or $\mathrm{D}$ position to simulate. Thus, the Nd concentration is $1.25 \%$ atomic ratio for a $2 \times 2 \times 2$ super cell model. The DOS of $\mathrm{BiFeO}_{3}$ and $1.25 \%$ atomic ratio $\mathrm{Nd}$ doped $\mathrm{BiFeO}_{3}$ were calculated also. The optical absorption coefficient was obtained by using the equation $\alpha=\sqrt{2 \mu_{o}} w \sqrt{\sqrt{\varepsilon_{1}^{2}(w)+\varepsilon_{2}^{2}(w)}-\varepsilon_{1}(w)},{ }^{43}$ which can be used in a nonconducting dispersive medium. Here $w$ is photon frequency, $\mu_{\mathrm{o}}$ is the permeability of free space, $\varepsilon_{1}$ and $\varepsilon_{2}$ are frequency-dependent real and imaginary parts of dielectric permittivity from the VASP output of the first-principle calculation.

\section{Results and Discussion}

The optical band gap depends strongly on the proper choice of the Hubbard parameter $\mathrm{U}$ for $\mathrm{Fe}^{41}$. It was found that when $\mathrm{U}=7.5 \mathrm{eV}$, there was the smallest deviation between experimental and calculated band gaps with $\mathrm{J}=0.9 \mathrm{eV}$ as shown in Table S1. Figure 1 shows the powder XRD spectra of BFONd and Nd concentration vs. $\chi^{2}$ (goodness of fit) from the Rietveld-refinement fitting curve (red line). The exsitence of a second phase of $\mathrm{Bi}_{2} \mathrm{Fe}_{4} \mathrm{O}_{9}$ resulted in a Nd-rich situation, as was expected initally. In order to find the $\mathrm{Nd}$ concerntration, we uesd the Rietveld refinement to calculate the least squared $\chi^{2}$ as a function of $\mathrm{Nd}$ concentration [Nd]. The calculated $\mathrm{Nd}$ concerntration $[\mathrm{Nd}]$ corresponding to the minimum $\chi^{2}$ occurrs at $\sim 1.25 \%$ (Fig. 1). The fractional coordinates and occupancies of the $\mathrm{Bi}, \mathrm{Fe}, \mathrm{O}$, and $\mathrm{Nd}$ atoms at the minimum, $\chi^{2} \sim 1.625$, are given in Table 1 . It is important to know that $\mathrm{Nd}$ atom may either substitute the $\mathrm{Bi}$-site or appear at the interstitial sites, as illustrated in Fig. 2 . Three interstitial-site cases (B, C, and D) have been considered. The Rietveld refinements indicate negative occupancies in these Nd interstitial sites, as given in Table S2, suggesting that Nd substitution does not occupy interstitial sites.

The dark-field STEM images and simulation along the [111] zone axis are given in Fig. 3 . The angles of the simulated diffraction pattern are the same as the experimental diffraction pattern, and $\mathrm{d}$ spacings are in the 5\% deviation range (Fig. 3(b)). The STEM image simulation in Fig. 3(c) agrees with the actual STEM image, revealing a rhombohedral R3c space group with lattice parameters of $\mathrm{a}=\mathrm{b}=\mathrm{c}=5.63447 \AA$ and $\alpha=59.3498^{\circ}$, as well as an $\mathrm{Nd}$ substitution of an $\mathrm{A}$-site $\mathrm{Bi}$ atom ${ }^{44}$. From the relaxation of first-principles calculation as shown in Table 2, the total free energy of $\mathrm{A}(\mathrm{Bi})$-site $\mathrm{Nd}$ substitution with $1.25 \%[\mathrm{Nd}]$ in 80 atoms has the smallest energy. This indicated a more thermodynamic and stable structure compared with the substituted-Fe site or interstitial-site cases and 


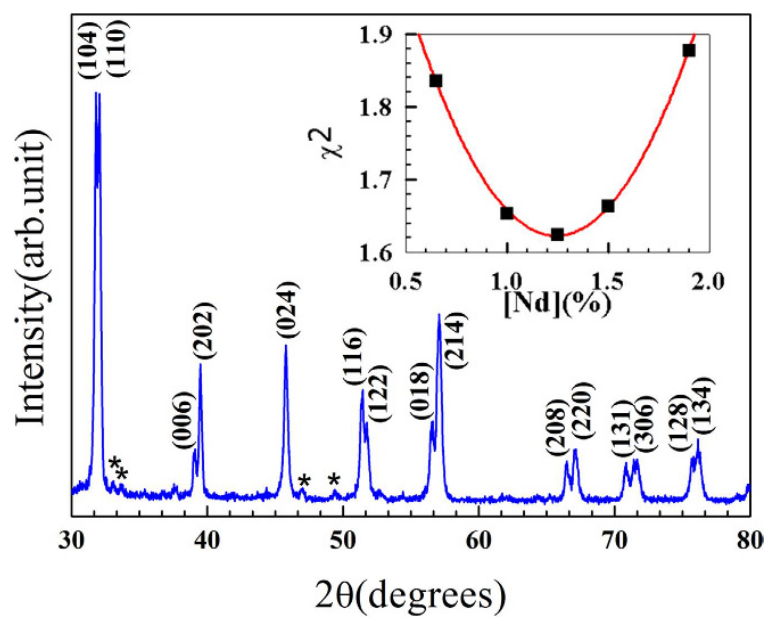

Figure 1. Powder XRD spectra of BFONd. The inset is the plot of $\mathrm{Nd}$ concentration vs. $\chi^{2}$ with a fitting curve (red line) and the minimum $\chi^{2}$ occurs at Nd concentration $\sim 1.25 \%$.

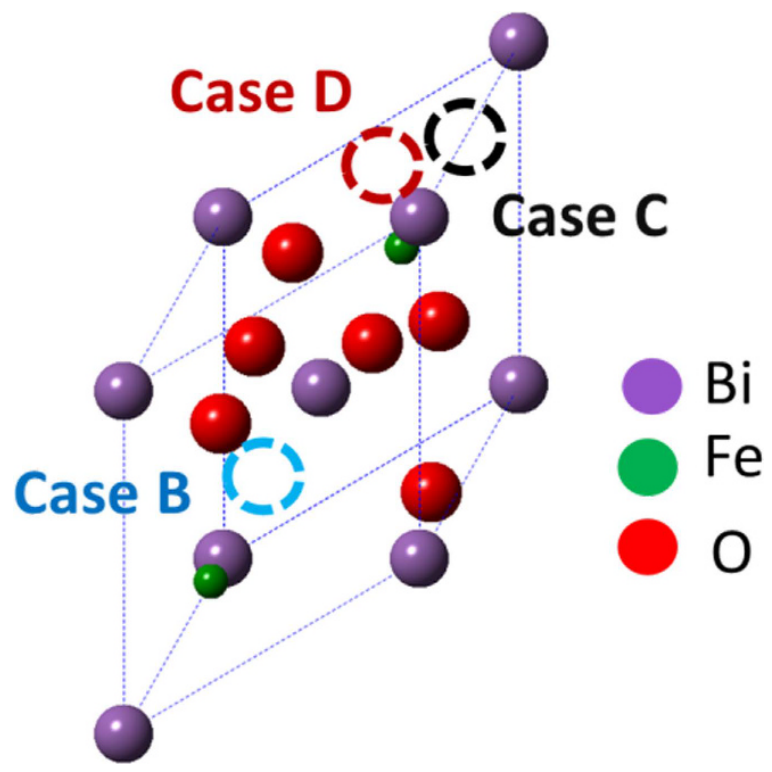

Figure 2. Coordinate interstitial sites of B, C, and D: $(0.360385,0.3608385,0.360385),(0.851385,0.851385$, $0.851385)$, and $(0.8006925,0.8006925,0.9256925)$.

\begin{tabular}{|l|c|c|c|c|}
\hline $\begin{array}{l}\text { A-site case at } \\
\chi^{2}=1.625\end{array}$ & \multicolumn{3}{|c|}{ Fractional coordinates } & Occupancy \\
\hline $\mathrm{Bi}$ & 0.000000 & 0.000000 & 0.003391 & 0.9375 \\
\hline $\mathrm{Fe}$ & 0.000000 & 0.000000 & 0.226548 & 1.0000 \\
\hline $\mathrm{O}$ & 0.444678 & 0.027363 & 0.961068 & 1.0000 \\
\hline $\mathrm{Nd}$ & 0.000000 & 0.000000 & 0.988694 & 0.0625 \\
\hline
\end{tabular}

Table 1. Fractional coordinates and occupancies for the A-site substitution.

with lattice parameters of $5.6843 \AA$ (super cell $11.3686 \AA$ ) and $\theta=59.243^{\circ}$. These results are consistent with the Rietveld-refinement analysis and the STEM image.

Figure 4(a) shows the ceramic disk with ITO electrodes used for photovoltaic (PV) measurements, the wavelength-dependent optical transmission of the ITO thin film, and the bright-field TEM image near the interface region between ITO film and the BFONd ceramics. The optical transmission of the ITO film is about $80 \%$ at an irradiation of $\lambda=405 \mathrm{~nm}$. Figure 4 (b) illustrates the open-circuit voltage $\left(\mathrm{V}_{\mathrm{oc}}\right)$ and the short-circuit current density $\left(\mathrm{J}_{\mathrm{sc}}\right)$ as irradiation was sequentially switched on and off with increasing irradiation intensity (I) for BFO 
(a)

(b)

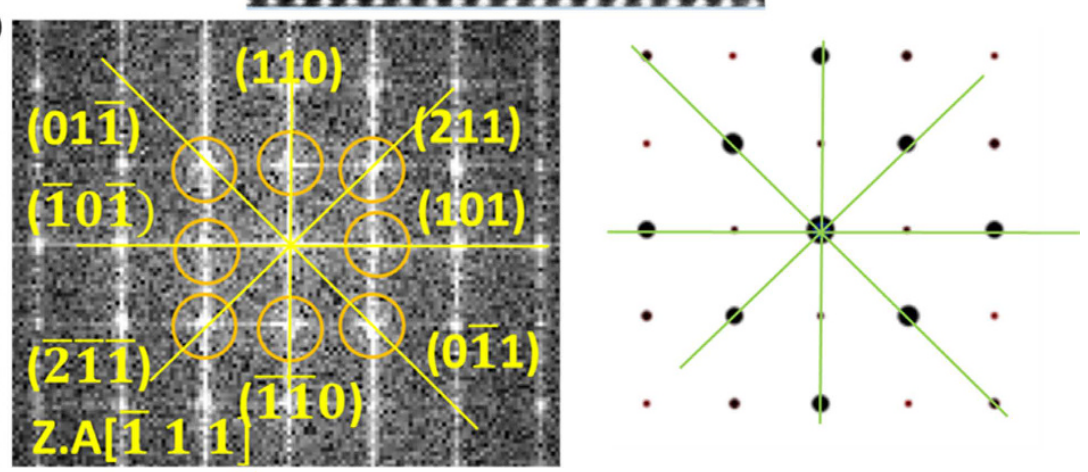

(c)
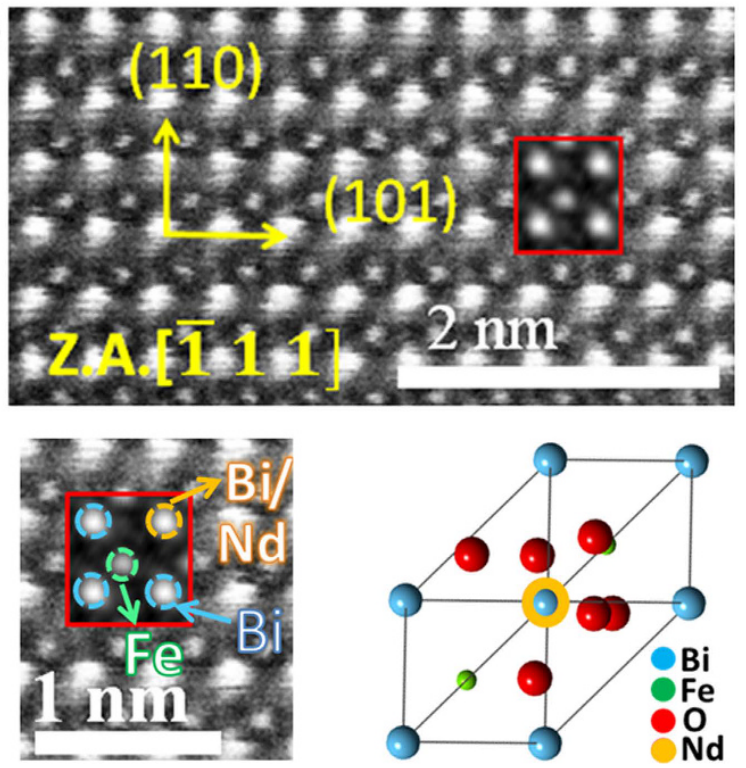

Figure 3. (a) Dark-field STEM image and diffraction pattern by fast Fourier transform for BFONd, (b) diffraction pattern and simulation along the[1111] zone axis, (c) dark-field STEM image simulation consistent with experiment image along the $[\overline{1} 11]$ zone axis.axis.

\begin{tabular}{|l|c|c|c|c|}
\hline & A-site & $\begin{array}{c}\text { interstitial } \\
\text { site B }\end{array}$ & $\begin{array}{c}\text { interstitial } \\
\text { site C }\end{array}$ & $\begin{array}{c}\text { interstitial } \\
\text { site D }\end{array}$ \\
\hline $\begin{array}{l}\text { Total } \\
\text { free } \\
\text { energy } \\
(\mathrm{eV})\end{array}$ & -505.1281 & -504.4567 & -504.2697 & -504.4567 \\
\hline
\end{tabular}

Table 2. Total free energies of $\mathrm{A}$-site (Bi-site) $\mathrm{Nd}$ substitution and interstitial sites ( $\mathrm{B}, \mathrm{C}$, and $\mathrm{D}$ cases). 
(a)

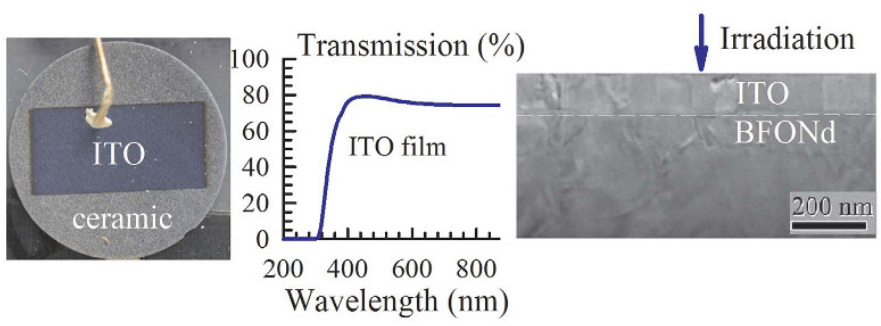

(b)

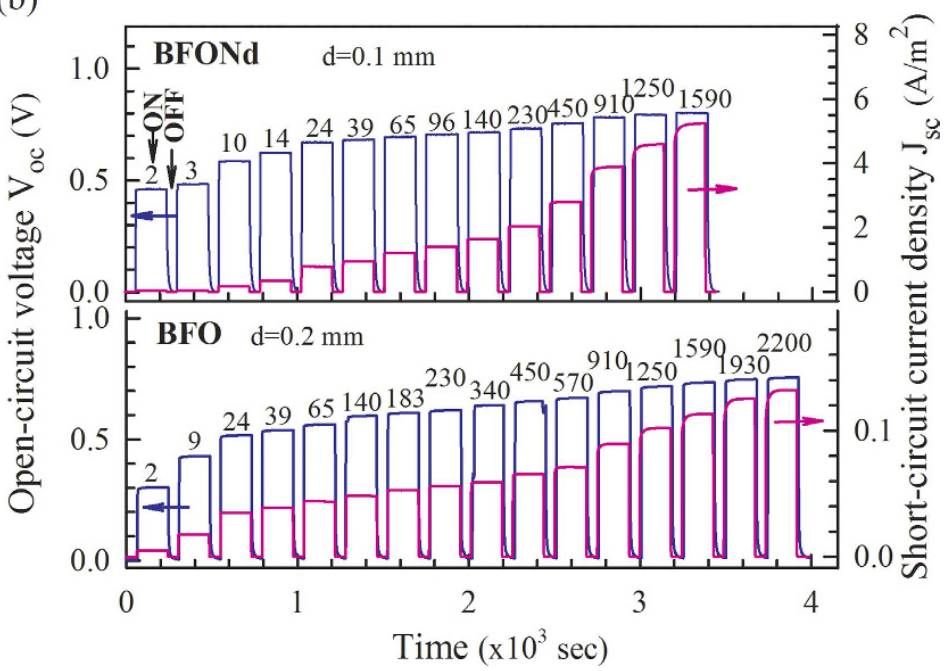

Figure 4. (a) Ceramic disk with ITO electrode, optical transmission of ITO film, and bright-field TEM image near the interface between ITO film and ceramic. (b) Open-circuit voltage $V_{o c}$ and short-circuit current density $\mathrm{J}_{\mathrm{sc}}$ as light was switched on and off with increasing irradiation intensity (in unit of $\mathrm{W} / \mathrm{m}^{2}$ ) labeled on the tops of illuminations.

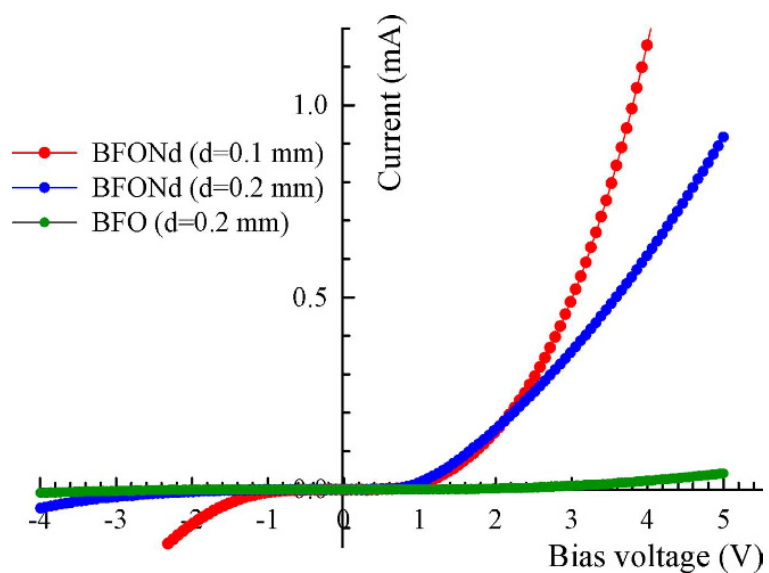

Figure 5. Characteristic curve of current vs. voltage (I-V) without irradiation.

(thickness $\mathrm{d}=0.2 \mathrm{~mm}$ ) and BFONd (thickness $\mathrm{d}=0.1 \mathrm{~mm}$ ). The characteristic curves of the current vs. the bias voltage were measured without irradiation (Fig. 5), suggesting a p-n-junction-like behavior between the ITO thin film and BFO (or BFONd) ceramics. As shown in Fig. 6(a), the power-conversion efficiency $\left(\eta=P_{\text {out }} / P_{\text {in }}\right)$ of BFONd with an optimized thickness $\mathrm{d}=0.1 \mathrm{~mm}$ can reach $\sim 0.82 \%$, the highest among major ferroelectric and BFO materials with varying amounts of electrodes ${ }^{13,30-32}$. For sample thickness of $d=0.2 \mathrm{~mm}$, the $\eta$ in BFONd is 19 times higher than that of BFO. A detailed comparison of photovoltaic studies in major perovskite ferroelectrics and p-type BFO materials can be found in our previous study ${ }^{13}$. In this work, the ITO/BFONd/Au heterostructure has demonstrated comparable photovoltaic effects, including open-circuit voltage $\left(V_{o c}\right)$, short-circuit current density (Jsc), and power-conversion efficiency ( $\eta$ ).

Figure S1 shows the optical transmission spectra of BFO and BFONd as a function of irradiation wavelength. The curves of $(\alpha h \nu)^{2}$ vs. h $\nu$ (photon energy) for BFO (red circles) and BFONd (blue circles) are plotted in 

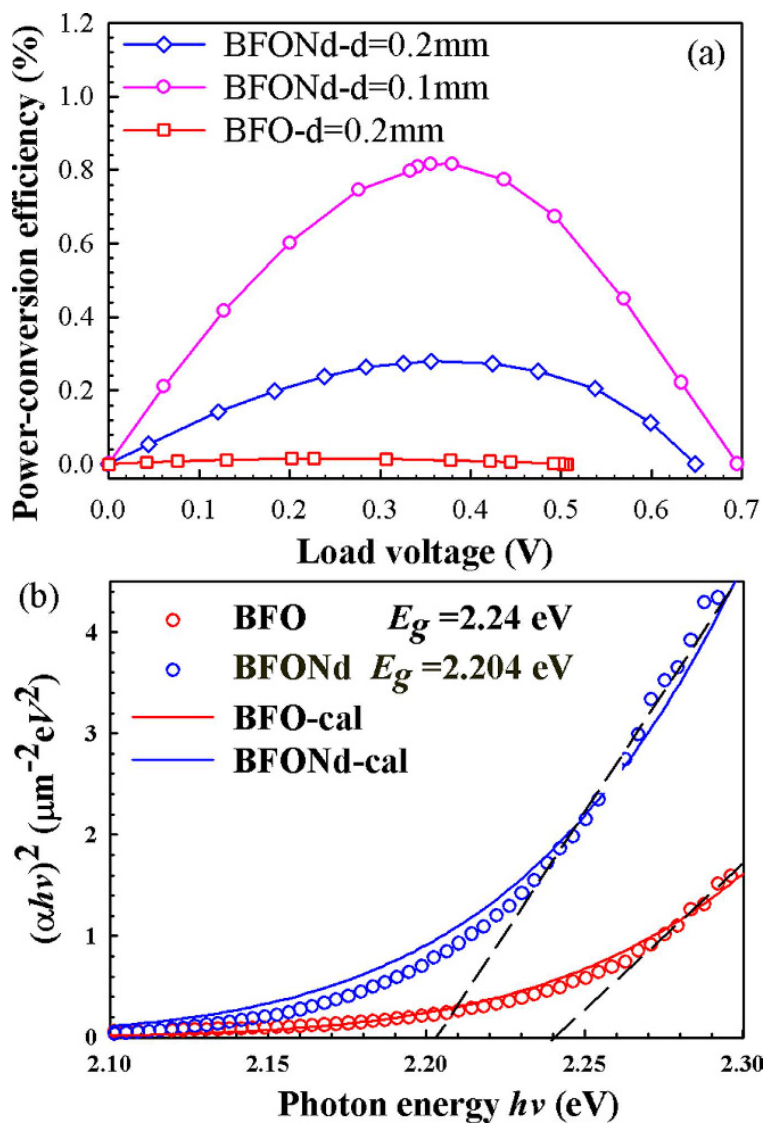

Figure 6. (a) Power conversion efficiency $(\eta)$ of BFONd (pink line with thickness $d=0.1 \mathrm{~mm}$ and blue line with $\mathrm{d}=0.2 \mathrm{~mm}$ ) and BFO (red line with $\mathrm{d}=0.2 \mathrm{~mm})$. The maximal $\eta$ values for BFO $(\mathrm{d}=0.2 \mathrm{~mm}), \mathrm{BFONd}$ $(\mathrm{d}=0.1 \mathrm{~mm})$ and BFONd $(\mathrm{d}=0.2 \mathrm{~mm})$ are respectively $0.015 \%, 0.816 \%$, and $0.279 \% .(\mathrm{b})(\alpha \mathrm{h} \nu)^{2} \mathrm{vs} . \mathrm{h} \nu$ (photon energy) for BFO (red circles) and BFONd (blue circles), where solid lines are the calculation result.

Fig. 6(b), where circles represent experimental data and solid lines represent the calculated results. As indicated by the dashed lines in Fig. 6(b), the experimental band gaps of BFO and BFONd are respectively about $2.24 \mathrm{eV}$ and $2.20 \mathrm{eV}$. The first-principles density functional calculation suggested that oxygen vacancies in BFO can shift the optical absorption to lower energy $y^{45,46}$. The different band gaps between this study and earlier reports ${ }^{3-8}$ may be associated with oxygen vacancies.

To explain the enhanced photovoltaic effects in the ITO/BFONd/Au heterostructure, the band structures, DOS, and projection density of states (PDOS) of BFO and BFONd in the rhombohedral $R 3 c$ structure are given in Fig. S2, indicating direct band gaps of $2.25 \mathrm{eV}$ and $2.23 \mathrm{eV}$ for $\mathrm{BFO}$ and $\mathrm{BFONd}$, respectively. The computed Brillouin zone path is $\Gamma(0,0,0) ; \mathrm{F}(1 / 2,1 / 2,0) ; \mathrm{L}(1 / 2,0,0) ; \mathrm{Z}(1 / 2,1 / 2,1 / 2)$ for the rhombohedral $R 3 c$ structure $^{47}$.

The calculated band gaps with $\mathrm{U}=7.5 \mathrm{eV}$ are consistent with the measured values. The A site $\mathrm{Nd}$ doping causes a reduction of the band gap from $E_{\mathrm{g}} \sim 2.25 \mathrm{eV}$ in $\mathrm{BFO}$ to $E_{\mathrm{g}} \sim 2.23 \mathrm{eV}$ in BFONd. From the density of states for BFO in Fig. S2(a), the spin-up and spin-down configurations exhibit symmetric distributions, confirming an antiferromagnetic tendency as suggested by the linear hysteresis curve of magnetization vs. the magnetic field in $\mathrm{BFO}^{48}$. As evidenced in Fig. S2(b), the DOS of BFONd exhibits an asymmetric configuration, which may be the reason for enhanced magnetic properties in some doped BFO materials ${ }^{21,22}$.

According to Eqs (1) and (2) for the smaller band gap $E_{\mathrm{g}}$ in BFONd, a larger $\alpha$ and photocurrent density can be expected in BFONd when compared with BFO. The correlation of the open-circuit voltage $\left(\mathrm{V}_{\mathrm{oc}}\right)$, photocurrent density $\left(\mathrm{J}_{\mathrm{ph}}\right)$, and $E_{\mathrm{g}}$ can be expressed by ${ }^{49}$.

$$
J_{\mathrm{ph}}=q w B N_{\mathrm{c}} N_{\mathrm{v}} \exp \left[\left(\mathrm{q} V_{\mathrm{oc}}-E_{\mathrm{g}}\right) / k \mathrm{~T}\right]
$$

where $\mathrm{w}$ is ceramic thickness, $\mathrm{B}$ is the radiative recombination factor which is temperature-dependent ${ }^{50}$, $\mathrm{q}$ is carrier charge, $T$ is room temperature $(300 \mathrm{~K}), k$ is the Boltzmann constant, and $\mathrm{N}_{\mathrm{c}}$ and $\mathrm{N}_{\mathrm{v}}$ are effective densities of states in the conduction and valence bands. These bands can be correlated to intrinsic carrier concentration $\left(n_{i}\right)$, i.e. ${ }^{51,52}$

$$
n_{i}^{2}=N_{c} N_{v} \exp \left(\frac{-E_{g}}{k T}\right)
$$


where the reduction of $E_{\mathrm{g}}$ due to $\mathrm{Nd}$ doping in the exponential term is the most important factor for the change in carrier concentration $\left(n_{\mathrm{i}}\right) . \mathrm{N}_{\mathrm{c}} \mathrm{N}_{\mathrm{v}}$ may be associated with current leakage and non-radiative recombination ${ }^{53-55}$, and we assume that $\mathrm{N}_{\mathrm{c}}$ and $\mathrm{N}_{\mathrm{v}}$ are dopant-independent parameters. (One $\mathrm{Nd}$ atom contributes 6 more valence electrons than the Bi atom in BFO.) Fig. S3(a) shows the open-circuit voltage $\mathrm{V}_{\text {oc }}$ and short-circuit current density $\left(\mathrm{J}_{\mathrm{sc}}\right)$ as functions of irradiation intensity from the ITO/BFO/Au and ITO/BFONd/Au heterostructures under irradiation for the same thickness of $\mathrm{d}=0.2 \mathrm{~mm} . \mathrm{V}_{\mathrm{oc}}$ and $\mathrm{J}_{\mathrm{sc}}$ increase rapidly in the low-intensity region, approaching saturation as irradiation intensifies. Since the dark current density $\left(\mathrm{J}_{\mathrm{dark}}\right)$ is smaller than the photocurrent density $\left(\mathrm{J}_{\mathrm{ph}}\right)$, the approximation of $\mathrm{J}_{\mathrm{sc}}=\mathrm{J}_{\mathrm{ph}}-\mathrm{J}_{\mathrm{dark}} \sim \mathrm{J}_{\mathrm{ph}}$ can be expected. We used Eq. (3) to estimate the $\mathrm{J}_{\mathrm{ph}}$ ratio between $\mathrm{BFO}$ and BFONd, i.e.

$$
\frac{J_{p h-B F O N d}}{J_{p h-B F O}}=\exp \left[\frac{-q\left(V_{o c-B F O N d}-V_{o c-B F O}\right)}{k T}\right] \exp \left[\frac{E_{g-B F O}-E_{q}-B F O N d}{k T}\right]
$$

As listed in Table $\mathrm{S} 3$, the $\mathrm{J}_{\mathrm{sc}-\mathrm{BFONd}} / \mathrm{J}_{\mathrm{sc}-\mathrm{BFO}}$ ratio is positively correlated with irradiation intensity, indicating that the photovoltaic effects in BFONd were enhanced with increasing intensity. This is consistent with the reduction of leakage current in the rare-earth doped $\mathrm{BFO}^{18}$. In contrast, the $\mathrm{J}_{\mathrm{ph}-\mathrm{BFONd}} / \mathrm{J}_{\mathrm{ph}-\mathrm{BFO}}$ decreases with increasing irradiation intensity. There are two possible mechanisms for the deviation between the $\mathrm{J}_{\mathrm{sc}-\mathrm{BFONd}} / \mathrm{J}_{\mathrm{sc}-\mathrm{BFO}}$ and $\mathrm{J}_{\mathrm{ph}-\mathrm{BFONd}} / \mathrm{J}_{\mathrm{ph}-\mathrm{BFO}}$ ratios. First, as shown in Eq. (3), the photocurrent $\mathrm{J}_{\mathrm{ph}}$ is sensitive to leakage current ${ }^{56}$ and intermediate defect states ${ }^{17}$, which are expected to have more profound effects in BFO at lower irradiation intensities, as charged carriers can be trapped in the low-lying defect states below the conduction band ${ }^{17}$. In addition, lower leakage current in BFONd is expected to have higher $\mathrm{V}_{\text {oc }}$ according to the Schockley ${ }^{57}$ and Poole-Frenkel emission equation ${ }^{16}$. Therefore, the $\mathrm{J}_{\mathrm{ph}-\mathrm{BFONd}} / \mathrm{J}_{\mathrm{ph}-\mathrm{BFO}}$ ratio tends to be larger at lower irradiation intensities. Secondly, the non-radiative carrier recombination lifetime $\left(\tau_{\mathrm{r}}\right)$ depends on irradiation intensity, decreasing as intensity decreases. The relation between carrier density and recombination time can be expressed by ${ }^{58}$

$$
n(t)=G \tau_{\mathrm{r}} \exp \left(-\mathrm{t} / \tau_{\mathrm{r}}\right)
$$

where $\mathrm{n}(\mathrm{t})$ is carrier concentration, $\mathrm{G}$ is the generation constant, $\tau_{\mathrm{r}}$ is the carrier recombination lifetime, and $\mathrm{t}$ is the time after irradiation. According to Eqs (4) and (6), $\mathrm{N}_{\mathrm{c}} \mathrm{N}_{\mathrm{v}}$ and $n$ are expected to have larger values for longer $\tau_{\mathrm{r}}$ at lower irradiation intensities, in which the defect states may cause a reduction of photocurrents. At higher irradiation intensities, carrier concentration $n$ may reach saturation in both BFO and BFONd, resulting in similar ratios appearing in $\mathrm{J}_{\mathrm{sc}-\mathrm{BFONd}} / \mathrm{J}_{\mathrm{sc}-\mathrm{BFO}}$ and $\mathrm{J}_{\mathrm{ph}-\mathrm{BFONd}} / \mathrm{J}_{\mathrm{ph}-\mathrm{BFO}}$.

\section{Conclusions}

A-site $\mathrm{Nd}^{3+}$ doped $\mathrm{BiFeO}_{3}\left(\mathrm{Bi}_{0.9375} \mathrm{Nd}_{0.0625}\right) \mathrm{FeO}_{3}$ (or BFONd) coupled with ITO and Au electrodes has demonstrated a maximal photovoltaic power conversion of $\sim 0.82 \%$ while under irradiation of wavelength $\lambda=405 \mathrm{~nm}$. This is not only the highest in recent PV studies of BFO materials, but is also promising for future application toward photo-driven actuators and optical sensors. The I-V characteristic curve (without irradiation) exhibits a p-n-like behavior and suggests a p-n junction interface between the ITO thin film and BFO (or BFONd) ceramics. The first-principles calculated band gaps for $\mathrm{BFO}$ and $\mathrm{BFONd}$ are $2.25 \mathrm{eV}$ and $2.23 \mathrm{eV}$, which are consistent with the experimental band gaps of $\sim 2.24 \mathrm{eV}$ and $\sim 2.20 \mathrm{eV}$, respectively. The enhanced photovoltaic responses can be mainly attributed to two phenomena: the reduction of the band gap in BFONd and the increase in valence electrons of the $\mathrm{Nd}$ atom.

\section{References}

1. C. Ederer \& N. A. Spaldin. Weak ferromagnetism and magnetoelectric coupling in bismuth ferrite. Phys. Rev. B 71, 060401(R) (2005).

2. S. M. Young, F. Zheng \& A. M. Rappe. First-Principles Calculation of the Bulk Photovoltaic Effect in Bismuth Ferrite. Phys. Rev. Lett. 109, 236601 (2012).

3. S. Y. Yang et al. Photovoltaic effects in $\mathrm{BiFeO}_{3}$. Appl. Phys. Lett. 95, 062909 (2009)

4. T. Choi et al. Switchable Ferroelectric Diode and Photovoltaic Effect in $\mathrm{BiFeO}_{3}$. Science 324, 63-66 (2009).

5. S. R. Basu et al. Photoconductivity in $\mathrm{BiFeO}_{3}$ thin films. Appl. Phys. Lett. 92, 091905 (2008).

6. X. S. Xu et al. Optical properties and magnetochromism in multiferroic $\mathrm{BiFeO}_{3}$. Phys. Rev. B 79, 134425 (2009).

7. A. Anshul et al. Photoluminescence and time-resolved spectroscopy in multiferroic $\mathrm{BiFeO}_{3}$ : effects of electric fields and sample aging. Appl. Phys. Lett. 102, 222901 (2013).

8. X. Chen et al. Optical and photoluminescence properties of $\mathrm{BiFeO}_{3}$ thin films grown on ITO-coated glass substrates by chemical solution deposition. Phys. Status Solidi A 209, 1456-60 (2012).

9. S. J. Clark \& J. Robertson. Band gap and Schottky barrier heights of multiferroic $\mathrm{BiFeO}_{3}$. Appl. Phys. Lett. 90, 132903 (2007).

10. M. Dawber, K. M. Rabe \& J. F. Scott. Physics of thin-film ferroelectric oxides. Rev. Mod. Phys. 77, 1083-1130 (2005).

11. W. Ji, K. Yao \& Y. C. Liang. Evidence of bulk photovoltaic effect and large tensor coefficient in ferroelectric $\mathrm{BiFeO}_{3}$ thin film. Phys. Rev. B 84, 094115 (2011).

12. S. Y. Yang et al. Above-bandgap voltages from ferroelectric photovoltaic devices. Nat Nano 5, 143-147 (2010).

13. C.-S. Tu et al. Enhanced photovoltaic effects in A-site samarium doped $\mathrm{BiFeO}_{3}$ ceramics: The roles of domain structure and electronic state. J. Eur. Ceram. Soc. 36, 1149-1157 (2016).

14. H. Yang et al. Rectifying current-voltage characteristics of $\mathrm{BiFeO}_{3} / \mathrm{Nb}$-doped $\mathrm{SrTiO}_{3}$ heterojunction. Appl. Phys. Lett. 92, 102113 (2008).

15. Q. Xu et al. The role of $\mathrm{Bi}$ vacancies in the electrical conduction of $\mathrm{BiFeO}_{3}$ : a first-principles approach. Dalton Trans. 43, 10787-10793 (2014).

16. G. W. Pabst, L. W. Martin, Y.-H. Chu \& R. Ramesh. Leakage mechanisms in $\mathrm{BiFeO}_{3}$ thin films. Appl. Phys. Lett. 90, 072902 (2007).

17. A. J. Hauser et al. Characterization of electronic structure and defect states of thin epitaxial $\mathrm{BiFeO}_{3}$ films by UV-visible absorption and cathodoluminescence spectroscopies. Appl. Phys. Lett. 92, 222901 (2008).

18. J.-H. Lee et al. Variations of ferroelectric off-centering distortion and $3 d$-4p orbital mixing in La-doped $\mathrm{BiFeO}_{3}$ multiferroics. Phys. Rev. B 82, 045113 (2010). 
19. X. H. Dai et al. Investigation of Leakage Current Behavior of $\mathrm{Pt} / \mathrm{Bi}_{0.975} \mathrm{La}_{0.025} \mathrm{Fe}_{0.975} \mathrm{Ni}_{0.025} \mathrm{O}_{3} / \mathrm{Pt}$ capacitor measured at different temperatures. Surf. Rev. Lett. 21, 1450029 (2014).

20. S. K. Singh, K. Maruyama \& H. Ishiwara. Reduced leakage current in La and Ni codoped $\mathrm{BiFeO}_{3}$ thin films. Appl. Phys. Lett. 91, 112913 (2007).

21. F. Huang et al. Effect of $\mathrm{Nd}$ dopant on magnetic and electric properties of $\mathrm{BiFeO}_{3}$ thin films prepared by metal organic deposition method. Appl. Phys. Lett. 89, 242914 (2006).

22. G. L. Yuan et al. Structural transformation and ferroelectromagnetic behavior in single-phase $\mathrm{Bi}_{1-x} \mathrm{Nd}_{x} \mathrm{FeO}_{3}$ multiferroic ceramics, Appl. Phys. Lett. 89, 052905 (2006).

23. A. Lahmara et al. Off-stoichiometry effects on $\mathrm{BiFeO}_{3}$ thin films. Solid State Ionics 202, 1-5 (2011).

24. C.-S. Tu et al. A-site strontium doping effects on structure, magnetic, and photovoltaic properties of $\left(\mathrm{Bi}_{1-x} \mathrm{Sr}_{x}\right) \mathrm{FeO}_{3-\delta}$ multiferroic ceramics. Ceram. Int. 41, 8417-8424 (2015).

25. Y. Ukai, S. Yamazaki, T. Kawae \& A. Morimoto. Polarization-induced photovoltaic effects in Nd-Doped $\mathrm{BiFeO}_{3}$ ferroelectric thin films. Jpn. J. Appl. Phys. 51, 09LE10 (2012).

26. C. S. Tu et al. Calcium-doping effects on photovoltaic response and structure in multiferroic $\mathrm{BiFeO}_{3}$ ceramics. J. Appl. Phys. 114, 124105 (2013).

27. Y. Yuan, Z. Xiao, B. Yang \& J. Huang. Arising applications of ferroelectric materials in photovoltaic devices. J. Mater. Chem. A 2, 6027-6041 (2014).

28. A. M. Glass, D. von der Linde \& T. J. Negran. High-voltage bulk photovoltaic effect and the photorefractive process in LiNbO ${ }_{3}$. Appl. Phys. Lett. 25, 233 (1974).

29. Y. Xu \& M. Shen. Structure and optical properties of nanocrystalline $\mathrm{BiFeO}_{3}$ films prepared by chemical solution deposition. Mater. Lett. 62, 3600-2 (2008).

30. W. Caia et al. Photovoltaic enhancement based on improvement of ferroelectric property and band gap in Ti-doped bismuth ferrite thin films. J. Alloys Compd. 617, 240-246 (2014).

31. S. Gupta, M. Tomar \& V. Gupta. Ferroelectric photovoltaic properties of Ce and $\mathrm{Mn}$ codoped $\mathrm{BiFeO}_{3}$ thin film. J. Appl. Phys. 115, $014102(2014)$.

32. B. Chen et al. Effect of top electrodes on photovoltaic properties of polycrystalline $\mathrm{BiFeO}_{3}$ based thin film capacitors. Nanotechnology 22, 195201 (2011).

33. Y. Zang et al. Enhanced photovoltaic properties in graphene/polycrystalline $\mathrm{BiFeO}_{3} / \mathrm{Pt}$ heterojunction structure. Appl. Phys. Lett. 99, 132904 (2011).

34. L.-Y. Chang et al. Raman vibrations and photovoltaic conversion in rare earth doped $\left(\mathrm{Bi}_{0.93} \mathrm{RE}_{0.07}\right) \mathrm{FeO}_{3}(\mathrm{RE}=\mathrm{Dy}, \mathrm{Gd}, \mathrm{Eu}, \mathrm{Sm})$ ceramics. Ceram. Int. 42, 834-842 (2016).

35. A. C. Larson \& R. B. Von Dreele. General Structure Analysis System (GSAS). Los Alamos National Laboratory Report. LAUR 86-748 (2004).

36. P. E. Blöchl. Projector augmented-wave method. Phys. Rev. B 50, 17953 (1994).

37. G. Kresse \& J. Hafner. Ab initio molecular dynamics for liquid metals. Phys. Rev. B 47, 558(R) (1993)

38. G. Kresse \& J. Hafner. Ab initio molecular-dynamics simulation of the liquid-metal-amorphous-semiconductor transition in germanium. Phys. Rev. B 49, 14251 (1994).

39. S. L. Dudarev et al. Electron-energy-loss spectra and the structural stability of nickel oxide: An LSDA+U study. Phys. Rev. B 57, 1505 (1998).

40. B. Himmetoglu et al. Hubbard-corrected DFT energy functionals: The LDA+U description of correlated systems. Int. J. Quantum Chem 114, 14-49 (2014).

41. S. Ju et al. Electronic structure, linear, and nonlinear optical responses in magnetoelectric multiferroic material $\mathrm{BiFeO}_{3}$. J. Chem. Phys. 130, 214708 (2009).

42. A. I. Liechtenstein et al. Density-functional theory and strong interactions: Orbital ordering in Mott-Hubbard insulators. Phys. Rev. B 52, R5467(R) (1995).

43. D. J. Griffiths. Introduction to Electrodynamics. 4th Edition, Pearson Education (2013).

44. F. Kubel \& H. Schmid. Structure of a ferroelectric and ferroelastic monodomain crystal of the perovskite $\mathrm{BiFeO}_{3}$. Acta Cryst. B46, 698-702 (1990)

45. S. Ju \& T. Y. Cai. First-principles studies of the effect of oxygen vacancies on the electronic structure and linear optical response of multiferroic $\mathrm{BiFeO}_{3}$. Appl. Phys. Lett. 95, 231906 (2009).

46. S. J. Clark \& J. Robertson. Energy levels of oxygen vacancies in $\mathrm{BiFeO}_{3}$ by screened exchange. Appl. Phys. Lett. 94, 022902 (2009).

47. W. Setyawan \& S. Curtarolo. High-throughput electronic band structure calculations: Challenges and tools. Computational materials science 49, 299-312 (2010).

48. A. K. Pradhan et al. Magnetic and electrical properties of single-phase multiferroic $\mathrm{BiFeO}_{3}$. J. Appl. Phys. 97, 093903 (2005).

49. R. R. King et al. Bandgap Engineering in High-Efficiency Multijunction Concentrator Cells. International Conference on Solar Concentrators for the Generation of Electricity or Hydrogen (Scottsdale, Arizona, 1-5 May 2005).

50. T. Trupke et al. Temperature dependence of the radiative recombination coefficient of intrinsic crystalline silicon. J. Appl. Phys. 94, 4930 (2003).

51. D. A. Neamen. Semiconductor Physics and Devices Basic Principles, $4^{\text {th }}$ Edition, Raghu Srinivasan, New York (2012).

52. S. M. Sze \& M. K. Lee. Semiconductor Devices: Physics and Technology, 3rd Edition, John wiley \& Sons (2013).

53. C. M. Proctor \& T.-Q. Nguyen. Effect of leakage current and shunt resistance on the light intensity dependence of organic solar cells. Appl. Phys. Lett. 106, 083301 (2015).

54. A. J. Morfa et al. Time-of-flight studies of electron-collection kinetics in polymer: Fullerene bulk-heterojunction solar cells. Adv. Funct. Mater. 21, 2580-2586 (2011).

55. A. J. Breeze, Z. Schlesinger, S. A. Carter \& P. J. Brock. Charge transport in $\mathrm{TiO}_{2} / \mathrm{MEH}-\mathrm{PPV}$ polymer photovoltaics. Phys. Rev. B 64, $125205(2001)$.

56. F. Arca et al. Interface trap states in organic photodiodes. Scientific Reports 3, 1324 (2013).

57. Z. He et al. Simultaneous enhancement of open-circuit voltage, short-circuit current density, and fill factor in polymer solar cells. Adv. Mater. 23, 4636-4643 (2011).

58. M. Alexe. Local mapping of generation and recombination lifetime in $\mathrm{BiFeO}_{3}$ single crystals by scanning probe photoinduced transient spectroscopy. Nano Lett. 12, 2193-2198 (2012).

\section{Acknowledgements}

This work is supported by the Ministry of Science and Technology of Taiwan.

\section{Author Contributions}

Yi-Ting Peng/sample preparations, all simulations, writing the initial version. Shan-Haw Chiou/simulations of $1^{\text {st }}$ principles calculations. Ching-Hung Hsiao/TEM operation. Chi-Shun Tu/Sample preparation, photovoltaic measurements, modifying initial version. Chuenhou (Hao) Ouyang/Corresponding author: the design and operation of the whole paper, modifying initial version. All authors reviewed the manuscript. 


\section{Additional Information}

Supplementary information accompanies this paper at http://www.nature.com/srep

Competing Interests: The authors declare no competing financial interests.

How to cite this article: Peng, Y.-T. et al. Remarkably enhanced photovoltaic effects and first-principles calculations in neodymium doped $\mathrm{BiFeO}_{3}$. Sci. Rep. 7, 45164; doi: 10.1038/srep45164 (2017).

Publisher's note: Springer Nature remains neutral with regard to jurisdictional claims in published maps and institutional affiliations.

(c) (i) This work is licensed under a Creative Commons Attribution 4.0 International License. The images or other third party material in this article are included in the article's Creative Commons license, unless indicated otherwise in the credit line; if the material is not included under the Creative Commons license, users will need to obtain permission from the license holder to reproduce the material. To view a copy of this license, visit http://creativecommons.org/licenses/by/4.0/

(c) The Author(s) 2017 